\title{
A single HII region model of the strong interstellar scattering towards Sgr A*
}

\author{
Egid Sicheneder ${ }^{\star}$ and Jason Dexter $\dagger$ \\ Max Planck Institute for Extraterrestrial Physics, Giessenbachstr. 1, 85748 Garching, Germany
}

Accepted XXX. Received YYY; in original form ZZZ

\begin{abstract}
Until recently, the strong interstellar scattering observed towards the Galactic center (GC) black hole, Sgr A*, was thought to come from dense gas within the GC region. The pulse broadening towards the transient magnetar SGR J1745-2900 near Sgr A* has shown that the source of the scattering is instead located much closer to Earth, possibly in a nearby spiral arm. We show that a single HII region along the line of sight, $1.5-4.8 \mathrm{kpc}$ away from Earth with density $n_{e}$ of a few $\simeq 100 \mathrm{~cm}^{-3}$ and radius $R \simeq 1.8-3.2$ pc can explain the observed angular broadening of Sgr A*. Clouds closer to the GC overproduce the observed DM, providing an independent location constraint that agrees with that from the magnetar pulse broadening. Our model predicts that sources within $\lesssim 10 \mathrm{pc}$ should show the same scattering origin as the magnetar and Sgr A*, while the nearest known pulsars with separations $>20$ pc should not. The radio spectrum of Sgr $\mathrm{A}^{*}$ should show a cutoff from free-free absorption at $0.2 \lesssim \nu \lesssim 1$ GHz. For a magnetic field strength $B \simeq 15-70 \mu \mathrm{G}$, the HII region could produce the rotation measure of the magnetar, the largest of any known pulsar, without requiring the gas near Sgr A* to be strongly magnetised.
\end{abstract}

Key words: Galaxy: centre - pulsars: individual (J745-2900) — scattering - HII regions

\section{INTRODUCTION}

Interstellar scattering by electron density fluctuations along the line of sight blurs radio images and pulsar emission profiles (angular and temporal broadening). Certain lines of sight through the Galaxy show anomalously strong scattering, notably towards the Galactic center (GC) black hole, Sgr $A^{*}$, whose image has been broadened to a constant 1 $\mathrm{GHz}$ size of $\simeq 1$ arcsec over $\simeq 40$ years of observations (e.g., Backer 1978; Krichbaum et al. 1993; Lo et al. 1993, 1998; Shen et al. 2005; Bower et al. 2006, 2014b).

The origin of the strong scattering towards the GC remains uncertain. From the lack of free-free absorption of the Sgr A* spectrum, van Langevelde et al. (1992) argued that the scattering source should be located $\Delta \gtrsim 200 \mathrm{pc}$ from Sgr $A^{*}$. From a decrease in the number density of extragalactic background sources near Sgr A*, Lazio \& Cordes (1998) found a best fit location of $\Delta \simeq 50-300$ pc. Producing the large observed image of Sgr $\mathrm{A}^{*}$ from turbulent gas so close to the GC would require either extreme turbulent

\footnotetext{
* E-mail: egid@mpe.mpg.de

$\dagger$ jdexter@mpe.mpg.de
}

energy densities or a special scattering geometry (Lithwick 2002; Goldreich \& Sridhar 2006).

A prediction of this scattering model was that radio pulsars in the GC should be rendered undetectable due to the spread of arrival times of their pulses becoming longer than their pulse periods. Recently, a rare transient magnetar SGR J1745-2900 was discovered $\simeq 0.1 \mathrm{pc}(\simeq 2.5$ arcsec $)$ in projection from Sgr A* (Kennea et al. 2013; Mori et al. 2013). Radio pulsations were detected from this source down to $1 \mathrm{GHz}$ (Eatough et al. 2013), and the pulse broadening from scattering was measured to be $2-3$ orders of magnitude smaller than predicted (Spitler et al. 2014). In addition, the elliptical scatter-broadened image of J1745-2900 was found to be identical to that of Sgr A* in both size and orientation (Bower et al. 2014a). The combination of angular and temporal pulse broadening from the same object allowed an estimate of the location of the scattering along the line of sight, $\Delta=5.9 \pm 0.3 \mathrm{kpc}$, far from the GC. The chance alignment of such a scattering region, of size $\lesssim 0.5$ $\operatorname{deg}$ (van Langevelde et al. 1992), with Sgr A* and the GC is strange unless strong scattering has a common astrophysical origin.

HII regions have long been thought to produce strong interstellar scattering towards the Galactic plane (e.g., 
Litvak 1971; Little 1973; Dennison et al. 1984). Here we assess the physical conditions required to produce the observed scattering towards Sgr A* in terms of a simple model of turbulent, ionized gas in such an HII region (§2). We show that the model can explain the observed scattering properties for a typical size and electron density (§3). From the range of allowed cloud properties $(\S 4)$, we calculate its contribution to the RM of the magnetar and make predictions for the scattering locations of other GC pulsars and the low-frequency cutoff to the radio spectrum of Sgr A*.

\section{METHODS}

We use the thin screen approximation (e.g., Ishimaru 1977; Blandford \& Narayan 1985; van Langevelde et al. 1992) to calculate the angular broadening associated with a given spectrum of density fluctuations arising from an HII region.

\subsection{Thin screen scattering model}

Turbulent, free electrons scatter electromagnetic radiation strongly, since the electron density fluctuates. The electron density fluctuations are described by (Cordes et al. 1985),

$\left\langle\delta n_{e}^{2}\right\rangle=\int P_{\delta n_{e}}(\mathbf{q}) \mathrm{d} \mathbf{q}$,

with the Kolmogorov spectrum,

$P_{\delta n_{e}}(\mathbf{q})=C_{n}(x)^{2} \cdot \mathbf{q}^{-\frac{11}{3}} ; \quad$ for $\mathbf{q}_{0} \ll \mathbf{q} \ll \mathbf{q}_{1}$,

where $\mathbf{q}$ denotes the wavenumber, and $q_{1}=2 \pi / l_{1}$ and $q_{0}=$ $2 \pi / l_{0}$ correspond to the inner and outer scales of the turbulenct spectrum, $l_{1}$ and $l_{0}$. Assuming isotropic turbulence, the visibility $V(\rho ; L)$ is given by (van Langevelde et al. 1992),

$$
\begin{aligned}
V(\rho ; D) & =\exp \left(-D_{\boldsymbol{\Phi}} / 2\right), \\
D_{\boldsymbol{\Phi}}(\rho) & =8 \pi r_{e}^{2} \lambda^{2} \int_{0}^{D} \mathrm{~d} x \int \mathrm{d} q q\left[1-J_{0}(q \rho)\right] P_{\delta n_{e}}(q),
\end{aligned}
$$

where $\rho$ is the baseline length, $D$ is the distance from the observer to the source and $J_{0}(q \rho)$ a Bessel function. The phase structure function $D_{\Phi}$ specifies the statistical properties of the turbulent medium.

For $\rho<2 \pi / l_{1}=q_{1}$, the visibility has a Gaussian profile:

$$
\begin{aligned}
V(\rho ; D) & =\exp \left(-\rho^{2} / \rho_{C}^{2}\right), \\
\rho_{C} & =\left[6 \pi^{2} \lambda^{2} r_{e}^{2} \mathcal{L}(D) q_{1}^{\frac{1}{3}}\right]^{-\frac{1}{2}},
\end{aligned}
$$

as is observed for $\operatorname{Sgr} \mathrm{A}^{*}$. Here $r_{e}$ is the classical electron radius. The function $\mathcal{L}(D)$ takes into account the strength and position of the turbulent medium along the line of sight,

$\mathcal{L}(D)=\int_{0}^{D} C_{n}^{2}(x)\left(\frac{x}{D}\right)^{2} \mathrm{~d} x \simeq C_{n}^{2} \gamma^{2} H$,

where $x$ is the distance of the screen from the source and $\gamma \equiv \Delta / D$ the relative screen location. The second step comes from taking $C_{n}^{2}(x)$ to be constant across the HII region and assuming the cloud thickness $H$ is small $(H \ll D)$.

The apparent image size $\theta$ and $\rho_{C}$ are related by,
$\rho_{C}=\frac{\lambda \cdot \sqrt{2 \ln (2)}}{\pi \cdot \theta}$.

We express the structure constant in terms of the outer scale $l_{0}$ (Cordes et al. 1985),

$C_{n}^{2}=\frac{\delta n_{e}^{2}}{6 \pi}\left(\frac{2 \pi}{l_{0}}\right)^{2 / 3}$

Using the thin screen approximation and equations 6-9, we can write the image size as,

$\theta=\frac{2 \sqrt{\ln 2} r_{e} \lambda^{2} \delta n_{e} \gamma H^{1 / 2}}{l_{0}^{1 / 3} l_{1}^{1 / 6}}$

\subsection{An HII region as a thin scattering screen}

We consider a uniform distribution of free electrons contained in a single HII region, with a size given by its Strömgren radius $R_{S}$,

$R_{S}=\left(\frac{3 N_{L y}}{4 \pi \alpha_{H} n_{e}^{2}}\right)^{\frac{1}{3}} \simeq 4.7\left(\frac{n_{e}}{100 \mathrm{~cm}^{-3}}\right)^{-2 / 3} \cdot N_{L y, f}^{1 / 3} \mathrm{pc}$

for an ionizing photon rate $N_{L y}=5 \times 10^{49} N_{L y, f} \mathrm{~s}^{-1}$, scaled to a value appropriate for a bright $\mathrm{O}$ star. The scaling factor $N_{L y, f}$ is a model parameter, with $N_{L y, f}=1$ in the fiducial case. At the radius $R_{S}$, the photoionization is in equilibrium with the recombination, characterized by the volumetric recombination rate $\alpha_{H} \simeq 4 \times 10^{-13} \mathrm{~cm}^{-3} \mathrm{~s}^{-1}$.

We connect the properties of the turbulent fluctuations with the density and size of the HII radius by assuming the line of sight passes through a cloud thickness $H=4 / 3 R_{S}$, the average value for a line of sight through a sphere, and parameterize the fluctuations as $\delta n_{e}=f n_{e}$, with $f \leq 1$. We further assume an inner scale $l_{1}=10^{4} \mathrm{~km}$ (e.g., Wilkinson et al. 1994) and an outer scale comparable to the cloud radius: $l_{0}=f_{2} R_{S}$ with $f_{2} \leq 1$. With these scalings, we re-write equation 10 as,

$\theta \simeq 0.4 \gamma f_{2}^{-1 / 3} f\left(\frac{n_{e}}{100 \mathrm{~cm}^{-3}}\right)\left(\frac{R_{S}}{1 \mathrm{pc}}\right)^{1 / 6}\left(\frac{\lambda}{30 \mathrm{~cm}}\right)^{2}$ arcsec.

where $\gamma=\Delta / D$ is the relative screen location along the line of sight defined in terms of the screen-source $(\Delta)$ and total $(D)$ distance. The same cloud properties produce a larger image when located closer to the observer.

\subsection{Geometrical implications}

Due to scattering, different light rays reach the observer at different times. The width of the resulting broadened pulse depends on the image size and the thin screen location as(e.g., Cordes \& Lazio 1997),

$t_{\mathrm{PB}} \simeq \frac{D}{8 \cdot \ln (2) \cdot c} \frac{1-\gamma}{\gamma} \cdot \theta^{2} \mathrm{sec}$,

called pulse broadening. 


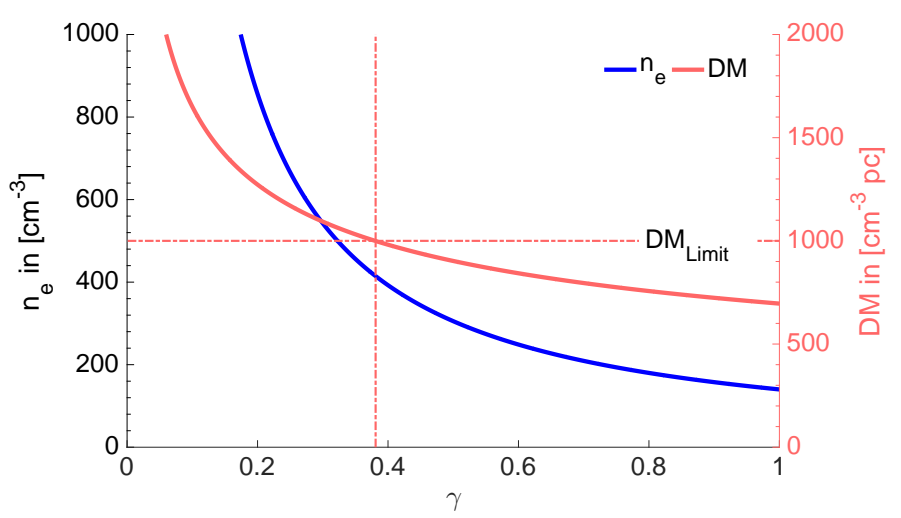

Figure 1. To account for the observed angular broadening of Sgr $\mathrm{A}^{*}$, the electron density (blue line and left axis) and dispersion measure (red line and right axis) must increase with decreasing distance between the model HII region and Galactic center. The horizontal line marks our assumed upper limit on the contribution to the dispersion measure of J1745-2900 from the HII region, $\mathrm{DM}_{\text {Limit }}=1000 \mathrm{pc} \mathrm{cm}^{-3}$, in turn constraining the screen location $\gamma \gtrsim 0.4$.

With a cloud size $R_{s}$ and location $\gamma$, our model gives the mean angular separation $\theta_{\text {sep }}$ of two sources, such that they can not be scattered by the same cloud:

$\theta_{\mathrm{sep}} \simeq \frac{R_{S}}{D-\Delta}=\frac{R_{S}}{D} \frac{1}{1-\gamma}$

$\theta_{\text {sep }} \simeq 0.033 \cdot \frac{N_{L y, f}^{\frac{1}{3}}}{1-\gamma}\left(\frac{n_{e}}{100 \mathrm{~cm}^{-3}}\right)^{-\frac{2}{3}} \mathrm{deg}$.

Second, the Earth rotates around the Galactic center at a speed $v_{\text {Orb }} \simeq 220 \mathrm{~km} / \mathrm{s}$. We can calculate in our framework how long it takes for the earth to pass the cloud, changing the observed scattering properties:

$t_{\mathrm{pass}}=\frac{R_{S}}{v_{O r b}} \frac{D}{D-\Delta}=\frac{R_{S}}{v_{\text {orb }}} \frac{1}{\gamma}$

$t_{\text {pass }}=21000 \cdot \frac{N_{L y, f}^{\frac{1}{3}}}{\gamma}\left(\frac{n_{e}}{100 \mathrm{~cm}^{-3}}\right)^{-\frac{2}{3}} \mathrm{yr}$.

\section{RESULTS}

We calculate the electron density $n_{e}$ and corresponding radius $R_{S}$ required to produce the observed angular broadening of Sgr $A^{*}$ and J1745-2900 $(\theta=945$ mas at $1 \mathrm{GHz}$, Bower et al. 2014a) from equation (12) as a function of the screen location $\gamma$ along the line of sight:

$$
\begin{aligned}
& n_{e} \simeq 140 \cdot f_{2}^{\frac{3}{8}} \cdot f^{-\frac{9}{8}} \cdot \gamma^{-\frac{9}{8}} \cdot N_{L y, f}^{-1 / 16} \mathrm{~cm}^{-3}, \\
& R_{S} \simeq 3.7 \cdot f^{\frac{3}{4}} \cdot f_{2}^{-\frac{1}{4}} \cdot N_{L y, f}^{3 / 8} \cdot \gamma^{\frac{3}{4}} \mathrm{pc}
\end{aligned}
$$

From the cloud density and radius, we calculate its contribution to the magnetar dispersion and rotation measures:

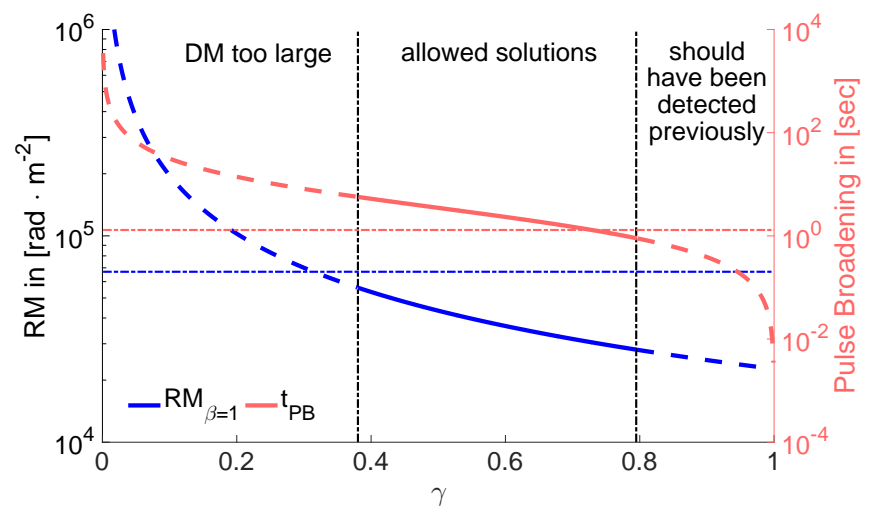

Figure 2. Rotation measure (RM, blue line and left axis) and pulse broadening (red line and right axis) versus the relative position of the HII region. Solid lines show the allowed solutions and the horizontal lines show the limits for DM and the pulse broadening. For clouds close to the GC $(\gamma \lesssim 0.4)$, the required densities become large, overproducing the observed DM and $\tau_{\mathrm{ff}}$. A cloud close to Earth $(\gamma \gtrsim 0.8)$ likely would have been detected already and so is not considered viable.

$$
\begin{aligned}
\mathrm{DM} & =\int_{0}^{L} n_{e}(x) \mathrm{d} x \\
& \simeq 620\left(\frac{n_{e}}{100 \mathrm{~cm}^{-3}}\right)^{1 / 3} \cdot N_{L y, f}^{1 / 3} \mathrm{pc} \mathrm{cm}^{-3} \\
\mathrm{RM} & =0.81 \int_{0}^{L} n_{e}(x) \cdot \mathbf{B} \mathrm{d} \mathbf{x} \\
& \simeq 5000 \cdot\left(\frac{n_{e}}{100 \mathrm{~cm}^{-3}}\right)^{1 / 3} \cdot\left(\frac{B_{\|}}{10 \mu \mathrm{G}}\right) \cdot N_{L y, f}^{1 / 3} \mathrm{rad} \mathrm{m}^{-2} \\
B_{\|} & \simeq 34 \cdot \beta^{-\frac{1}{2}} \cdot T_{e, 4}^{\frac{1}{2}} \cdot\left(\frac{n_{e}}{100 \mathrm{~cm}^{-3}}\right)^{1 / 2} \mu \mathrm{G}
\end{aligned}
$$

Using equation (18), we can re-write these in terms of the location $\gamma$ of the cloud:

$\mathrm{DM} \simeq 700 \cdot f_{2}^{\frac{1}{8}} \cdot f^{-\frac{3}{8}} \cdot \gamma^{-\frac{3}{8}} \cdot N_{L y, f}^{5 / 16} \mathrm{pc} \mathrm{cm}^{-3}$
$\mathrm{RM} \simeq 22,700 \cdot \beta^{-1 / 2} \cdot T_{e, 4}^{1 / 2} \cdot f_{2}^{\frac{5}{16}} \cdot f^{\frac{15}{16}} \cdot \gamma^{-\frac{15}{16}} \cdot N_{L y, f}^{9 / 32} \cdot \mathrm{rad} \mathrm{m}^{-2}$

where $\beta$ is the ratio of the thermal to magnetic pressure and $T_{e, 4}=T_{e} / 10^{4} \mathrm{~K}$ is the electron temperature. We set the parameters $f, f_{2}$ and $N_{L y, f}$ (introduced in section $\S 2.2$ ), equal to 1 , unless otherwise mentioned. Figure 1 shows the particle density $n_{e}$ and DM as a function of the screen location. Both quantities increase for screens closer to the GC, where producing the observed angular size requires large values of $C_{n}^{2}$.

We also calculate the free-free optical depth $\tau_{\text {ff }}$ (Rybicki \& Lightman 1979) through the cloud:

$$
\begin{aligned}
\tau_{\mathrm{ff}} & \simeq 0.024 \cdot T_{e, 4}^{-3 / 2} \cdot N_{L y, f}^{\frac{1}{3}} \cdot\left(\frac{n_{e}}{100 \mathrm{~cm}^{-3}}\right)^{4 / 3}\left(\frac{\nu}{1 \mathrm{GHz}}\right)^{-2} \\
& \simeq 0.038 \cdot f_{2}^{\frac{1}{2}} \cdot f^{-\frac{3}{2}} \cdot T_{e, 4}^{-\frac{3}{2}} \gamma^{-\frac{3}{2}} N_{L y, f}^{1 / 4},
\end{aligned}
$$

where we use $\nu=1 \mathrm{GHz}$ and set the Gaunt factor $g_{\text {ff }} \simeq 7$ 
for this frequency (Karzas \& Latter 1961). Finally, the geometric quantities are given as,

$$
\begin{aligned}
& \theta_{\text {sep }} \simeq 0.027 \cdot f_{2}^{-\frac{1}{4}} \cdot f^{\frac{3}{4}} \cdot \frac{\gamma^{\frac{3}{4}}}{1-\gamma} \cdot N_{L y, f}^{\frac{3}{8}} \mathrm{deg}, \\
& t_{\text {pass }}=16,800 \cdot f^{\frac{3}{4}} \cdot f_{2}^{-\frac{1}{4}} \cdot N_{L y, f}^{3 / 8} \cdot \frac{\gamma^{\frac{3}{4}}}{1-\gamma} \mathrm{yr} .
\end{aligned}
$$

We compare these model values to the measured quantities from the line of sight towards Sgr A* / J1745-2900. We use upper limits of DM $<1000 \mathrm{pc} \mathrm{cm}^{-3}, \tau_{\mathrm{ff}}<1$ at $1 \mathrm{GHz}$ (Melia \& Falcke 2001; Roy \& Pramesh Rao 2004), and lower limits of $t_{\text {pass }}>40 \mathrm{yr}, \theta_{\text {sep }}>2.5 \operatorname{arcsec}$ to constrain the allowed range of screen locations. This DM limit is smaller than the total value towards J1745-2900 $\left(\mathrm{DM}=1778 \mathrm{pc} \mathrm{cm}^{-3}\right.$ Eatough et al. 2013). We use it as a limit because it is comparable to both the observed DM values toward the nearest pulsars to the GC (Johnston et al. 2006; Deneva et al. 2009), and to the Galactic disc component of the DM along this line of sight in the NE2001 model (e.g., the $\Delta \mathrm{DM}$ between lines of sight with $l=0^{\circ}$ and $l=2^{\circ}$ with $b=0^{\circ}$, Cordes \& Lazio 2002).

Screens with $\gamma \lesssim 0.4$ exceed this DM limit, and so are excluded. A weaker limit $\gamma \gtrsim 0.1$ comes from the free-free optical depth towards Sgr A*. For all models, the time for the line of sight to move across the cloud is much longer than the 40 years over which the size of Sgr A* has been measured, and so we do not consider this constraint further. These constraints do not rule out large HII regions very close to Earth. Nearby HII regions should already have been detected, and so we restrict the allowed models to those with $D-\Delta>1.5 \mathrm{kpc}$.

Figure 2 shows the temporal broadening (red curve, right axis scale) associated with our thin screen model as a function of its location (equation 13). Bower et al. (2014a) combined angular and temporal broadening measurements of J1745-2900 (Spitler et al. 2014) to locate the screen location as $\Delta=5.9 \pm 0.3 \mathrm{kpc}$. Our constraints are independent of and consistent with this measurement of the scattering location.

The left hand axis and blue curve in figure 2 show the model RM for an equipartition strength magnetic field $(\beta=1)$. At $\gamma \simeq 0.4$ the model (see equation $(21)$ ) can explain even all of the observed $\mathrm{RM} \simeq 6.7 \cdot 10^{4} \mathrm{rad} \mathrm{m}^{-2}$ (Eatough et al. 2013) towards J1745-2900, the largest of any pulsar. The magnetic field strength at $\gamma \simeq 0.4$ is $B_{\|} \simeq 70 \mu \mathrm{G}$ (see equation (22) with $\beta=1$ ). Towards $\gamma \simeq 0.8$ the model $\mathrm{RM}$ and magnetic field strength drop, at least able to explain the bulk of the observed RM towards J1745-2900. Where the magnetic field strength is $B_{\|} \simeq 45 \mu \mathrm{G}$. This is further shown in figure 3 , which shows RM vs. $t_{\mathrm{PTD}}$ for our model with different assumed field strengths compared to the observed values from J1745-2900. Except for very low field strengths (e.g., Harvey-Smith et al. 2011), the allowed models contribute significantly to the observed RM.

\section{DISCUSSION}

The recently discovered GC magnetar SGR J1745-2900, $\simeq 0.1 \mathrm{pc}$ from $\mathrm{Sgr} \mathrm{A}^{*}$ in projection, shows the same scatterbroadened radio image as Sgr A*, but pulse broadening $2-3$

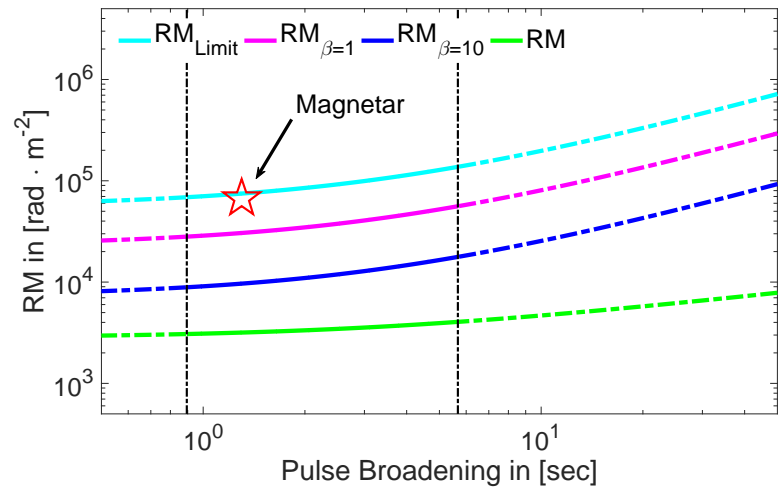

Figure 3. Rotation measure (RM) versus the pulse broadening. A magnetised cloud (cyan and purple lines) can produce the observed $\mathrm{RM} \simeq 6.7 \cdot 10^{4} \mathrm{rad} \mathrm{m^{-2 }}$ (red star, see (Eatough et al. 2013)) which was previously thought to require a dynamically important magnetic field in gas falling onto Sgr A* (Eatough et al. 2013).

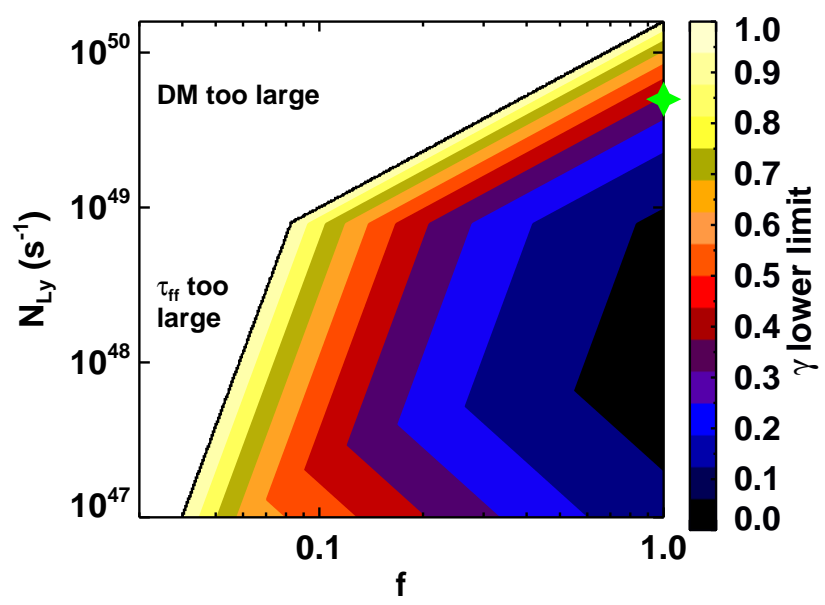

Figure 4. Lower limit on screen distance from the GC $(\gamma)$ as a function of the ionizing photon rate $N_{L y}$ and fluctuation strength $f$. The relevant constraints are $\mathrm{DM}<1000 \mathrm{pc} \mathrm{cm}^{-3}, \tau_{\mathrm{ff}}<1$ at $1 \mathrm{GHz}$, and $\theta_{\text {sep }}>2.5$ arcsec. The green star shows our fiducial parameter choices $f=1, N_{L y}=5 \times 10^{49} \mathrm{~s}^{-1}$. At large $N_{\mathrm{Ly}}$, low $f$ models overproduce the observed DM, while at low $N_{\mathrm{Ly}}$ the optical depth is too large. In the lower right region, the limit on $\gamma$ comes from forcing the cloud to be large enough to produce both the magnetar and Sgr A* images. The white region shows models where all $\gamma$ are excluded, placing a constraint $f \gtrsim 0.1$. The range of allowed screen locations we find is generic to a large part of the parameter space. Screens close to the GC $(\gamma \gtrsim 0.05)$ would require a weak ionizing source driving strong turbulence, and are only possible in a narrow range of parameter space.

orders of magnitude smaller than predicted (Bower et al. 2014a; Spitler et al. 2014). The combination of these measurements implies that the turbulent gas producing the observed image is located far from the GC.

HII regions have long been candidates for the observed strong interstellar scattering towards the Galactic plane. We have shown that for typical properties, $n_{e} \simeq 100 \mathrm{~cm}^{-3}, R_{S} \simeq$ 


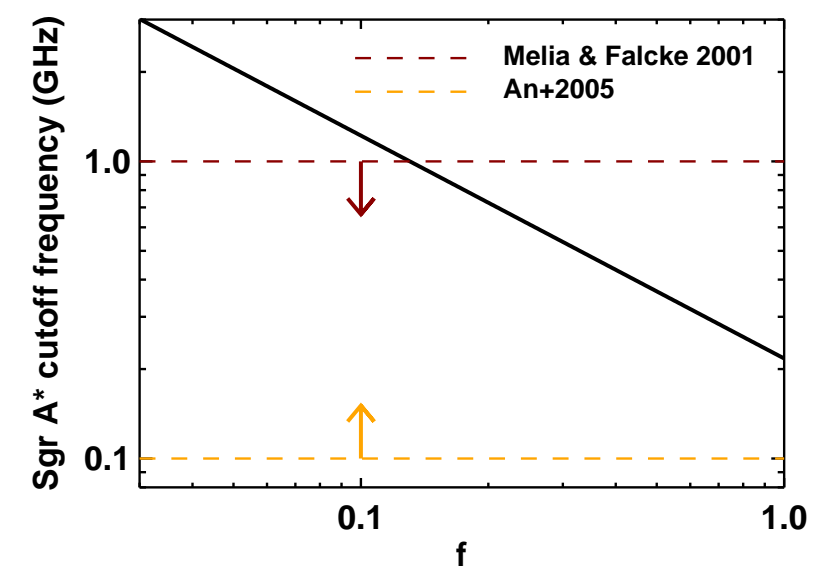

Figure 5. Predicted cutoff frequency (where $\tau_{\mathrm{ff}}=1$ ) for the radio spectrum of Sgr A* as a function of the fluctuation strength $f$, assuming a screen location $\gamma=0.75$ found by Bower et al. (2014a). Detections of Sgr $\mathrm{A}^{*}$ below $1 \mathrm{GHz}$ constrain $f \gtrsim 0.1$, while our model predicts a cutoff should be seen at a frequency above $\simeq 200 \mathrm{MHz}$, in agreement with low frequency spectral measurements (Nord et al. 2004; An et al. 2005).

$1.8-3.2 \mathrm{pc}$, such an HII region $1.5-4.8 \mathrm{kpc}$ from Earth can explain the angular broadening of Sgr A*. Placing the screen closer to the GC $(\gamma \lesssim 0.4)$ overproduces the observed DM of the magnetar, and for screens close to the GC $(\gamma \lesssim 0.1)$ also the free-free optical depth towards Sgr A*. This constraint on the location of the scattering medium is independent of, and consistent with, the geometric result $\gamma \simeq 0.75$ found by combining the angular and temporal broadening of the magnetar (equation 13, Bower et al. 2014a).

We have assumed a uniform HII region with a size and particle density related by the Strömgren radius for an assumed rate of ionizing photons, $N_{L y}=5 \times 10^{49} \mathrm{~s}^{-1}$, and fluctuation strength $\delta n_{e}=f n_{e}$ with $f=1$. Figure 4 shows how our lower limit on the screen location, $\gamma$, depends on these parameters. Decreasing the turbulent scaling, $f$, increases the required particle density to produce the angular broadening of Sgr $\mathrm{A}^{*}$. This in turn increases the DM and $\tau_{\text {ff }}$ at each location, and so requires the screen to be located farther from the GC. A similar effect results from increasing $N_{L y}$. This causes the HII region to be larger for fixed $\delta n_{e}$, and increases the DM since its weighting with $R$ is stronger than that of $C_{n}^{2}$. The combination of these effects means that the model is only compatible with the observed DM and $\tau_{\text {ff }}$ $(\gamma<1)$ for strong turbulence, $f \gtrsim 0.1$, and $N_{L y} \lesssim 10^{50} \mathrm{~s}^{-1}$. In models of MHD turbulence, it is sometimes assumed that $f \simeq \beta^{-1}$ (e.g., Goldreich \& Sridhar 2006). Our limit on $f$ could then favor a relatively large value of $B$, which in turn leads to a larger contribution of the HII region to the observed RM.

A successful model of scattering towards the magnetar also needs to account for its temporal broadening, $1.3 \pm 0.2 \mathrm{~s}$ at $1 \mathrm{GHz}$ (Spitler et al. 2014), which fixes $\gamma \simeq 0.75$ (Bower et al. 2014a) in a thin screen model. For this location, we find the following HII region properties: $n_{e} \simeq$ $200 \mathrm{~cm}^{-3}, R_{S} \simeq 3 \mathrm{pc}, \mathrm{DM} \simeq 800 \mathrm{pc} \mathrm{cm}^{-3}, \tau_{\mathrm{ff}} \simeq 0.05 \nu_{\mathrm{GHz}}^{-2}$, $\theta_{\text {sep }} \simeq 5$ arcmin. The nearest pulsars in angular separation are $10-15$ arcminutes from Sgr A* $(\simeq 25$ pc at the distance of the GC, Johnston et al. 2006; Deneva et al. 2009). All of these GC pulsars show large pulse broadening, and so measurements of their radio images can constrain the screen location $\gamma$ in the same fashion as done by Bower et al. (2014a) for SGR J1745-2900. A prediction of our model is that the single HII region is unlikely to cover all of these pulsars, so that their values of $\gamma$ should be different than that of the magnetar. This prediction is consistent with recent measurements of angular broadening for the other GC pulsars (Dexter et al., in prep.). Significant angular broadening of OH/IR stars is seen on larger scales $\lesssim 0.5 \mathrm{deg}$ (van Langevelde et al. 1992; Frail et al. 1994). In our model there would need to be multiple clouds covering the region. This is consistent with the variation of the maser angular sizes and the free-free optical depth to extragalactic background sources on these scales (Roy 2013).

Our model also predicts a low-frequency cutoff to the Sgr $A^{*}$ spectrum $\left(\tau_{\mathrm{ff}}=1\right)$ at $\gtrsim 0.2 \mathrm{GHz}$ (Figure 5 . The smallest values of the cutoff frequency occur for $f=1$, while values $f \lesssim 0.1$ are ruled out by the unbroken power law Sgr A* spectrum down to $1 \mathrm{GHz}$ (Melia \& Falcke 2001), showing again that strong turbulence is needed to explain the scattering towards Sgr A*. An et al. (2005) and Nord et al. (2004) find evidence for a break in the spectrum at wavelengths between $47-100 \mathrm{~cm}(100-866 \mathrm{MHz})$, consistent with our prediction. Measuring the low-frequency cut off shape of the Sgr A* spectrum would help to directly measure the fluctuation strength and further constrain the model.

The RM of the magnetar is an order of magnitude larger than that of any other pulsar, and for this reason was previously thought to come from gas local to the GC. To produce the observed RM, this gas would need to be threaded by very strong, uniform magnetic fields (Eatough et al. 2013). As an alternative, we show that for magnetic field strengths $\simeq 15-70 \mu \mathrm{G}$ a single HII region can produce much or all of the observed RM (figure 3). This field strength is large, but within the range of observed HII region values of both B (e.g., Heiles et al. 1981; Rodríguez et al. 2012) and $\beta$ (Harvey-Smith et al. 2011). Therefore we caution that the $\mathrm{RM}$ of the magnetar does not necessarily require that the gas near Sgr A* be highly magnetised. The HII region cannot however produce the order of magnitude larger RM seen towards Sgr A* itself, which is thought to arise within the surrounding accretion flow (Bower et al. 2003; Marrone et al. 2007).

Schnitzeler et al. (2016) measured the RM towards the other GC pulsars and found two others with $\mathrm{RM} \simeq$ $10^{4} \mathrm{rad} \mathrm{m}^{-2}$. If the very large RM for SGR J1745-2900 is produced from extremely strong, ordered fields within the central parsec, it seems strange that smaller but comparable RMs would be found for these other objects much further away. The mean field strength estimated from $\mathrm{RM} / \mathrm{DM} \simeq$ $30 \mu \mathrm{G}$ for these pulsars is similar to that of SGR J17452900, and so distant HII regions with mean field strengths like we require could be a more natural explanation. On the other hand, the RM towards the magnetar and other GC pulsars is an order of magnitude higher than for other known pulsars, while our model should apply to many heavily scattered lines of sight in the inner Galaxy. This suggests that other heavily scattered lines of sight either have weaker 
field strengths (e.g. the HII regions towards the GC would have to be uncomfortably "special"), are preferentially not detected by pulsar surveys (preventing detections of large RMs away from the GC), or that the GC environment on scales of tens of pc does in fact produce the large observed RMs as suggested by Schnitzeler et al. (2016).

In this scenario for the scattering towards $\operatorname{Sgr} \mathrm{A}^{*}$, the small HII region is aligned with $\mathrm{Sgr} \mathrm{A}^{*}$ by chance and does not cover the entire GC. The chance probability of this occurrence is small unless lines of sight with such strong scattering are common. A significant fraction $(\gtrsim 10 \%)$ of lines of sight through the inner Galaxy pass through a known HII region (Anderson et al. 2014), and gas with typical densities we find of $\sim 100 \mathrm{~cm}^{-3}$ is seen strongly in emission towards the inner Galaxy at the radial velocity corresponding to the Scutum spiral arm (Langer et al. 2016). A handful of lines of sight with very strong scattering are known (e.g., Rodriguez et al. 1982; Wilkinson et al. 1994) and many extragalactic background sources behind the Galactic plane are known to be heavily scattered (e.g., Lazio et al. 1999; Claussen et al. 2002; Beasley et al. 2002; Pushkarev \& Kovalev 2015). If such lines of sight are common, then HII regions as modeled here should contribute significantly to the observed DM and RM towards heavily scattered objects. Lower limits on the DM, and therefore revised pulsar distance estimates, can be inferred from our model in cases where the properties of the intervening HII region can be measured.

\section{ACKNOWLEDGEMENTS}

We thank E. Quataert, F. Eisenhauer, S. Gillessen, R. Herrera-Camus, G. Bower, and R. Wharton for useful discussions. This work was supported by a Sofja Kovalevskaja Award from the Alexander von Humboldt Foundation of Germany.

\section{REFERENCES}

An T., Goss W. M., Zhao J.-H., Hong X. Y., Roy S., Rao A. P., Shen Z.-Q., 2005, ApJ, 634, L49

Anderson L. D., Bania T. M., Balser D. S., Cunningham V., Wenger T. V., Johnstone B. M., Armentrout W. P., 2014, ApJS, 212, 1

Backer D. C., 1978, ApJ, 222, L9

Beasley A. J., Gordon D., Peck A. B., Petrov L., MacMillan D. S., Fomalont E. B., Ma C., 2002, ApJS, 141, 13

Blandford R., Narayan R., 1985, MNRAS, 213, 591

Bower G. C., Wright M. C. H., Falcke H., Backer D. C., 2003, ApJ, 588, 331

Bower G. C., Goss W. M., Falcke H., Backer D. C., Lithwick Y., 2006, ApJ, 648, L127

Bower G. C., et al., 2014a, ApJ, 780, L2

Bower G. C., et al., 2014b, ApJ, 790, 1

Claussen M. J., Goss W. M., Desai K. M., Brogan C. L., 2002, ApJ, 580, 909

Cordes J. M., Lazio T. J. W., 1997, ApJ, 475, 557

Cordes J. M., Lazio T. J. W., 2002, ArXiv Astrophysics e-prints,

Cordes J. M., Weisberg J. M., Boriakoff V., 1985, ApJ, 288, 221

Deneva J. S., Cordes J. M., Lazio T. J. W., 2009, ApJ, 702, L177

Dennison B., Thomas M., Booth R. S., Brown R. L., Broderick J. J., Condon J. J., 1984, A\&A, 135, 199
Eatough R. P., et al., 2013, Nature, 501, 391

Frail D. A., Diamond P. J., Cordes J. M., van Langevelde H. J., 1994, ApJ, 427, L43

Goldreich P., Sridhar S., 2006, ApJ, 640, L159

Harvey-Smith L., Madsen G. J., Gaensler B. M., 2011, ApJ, 736,83

Heiles C., Chu Y.-H., Troland T. H., 1981, ApJ, 247, L77

Ishimaru A., 1977, Appl. Opt., 16, 3190

Johnston S., Kramer M., Lorimer D. R., Lyne A. G., McLaughlin M., Klein B., Manchester R. N., 2006, MNRAS, 373, L6

Karzas W. J., Latter R., 1961, ApJS, 6, 167

Kennea J. A., et al., 2013, ApJ, 770, L24

Krichbaum T. P., et al., 1993, A\&A, 274, L37

Langer W. D., Goldsmith P. F., Pineda J. L., 2016, A\&A, 590, A43

Lazio T. J. W., Cordes J. M., 1998, ApJ, 505, 715

Lazio T. J. W., Anantharamaiah K. R., Goss W. M., Kassim N. E., Cordes J. M., 1999, ApJ, 515, 196

Lithwick Y., 2002, PhD thesis, California Institute of Technology

Little L. T., 1973, Astrophys. Lett., 13, 115

Litvak M. M., 1971, ApJ, 170, 71

Lo K. Y., Backer D. C., Kellermann K. I., Reid M., Zhao J. H., Goss W. M., Moran J. M., 1993, Nature, 362, 38

Lo K. Y., Shen Z.-Q., Zhao J.-H., Ho P. T. P., 1998, ApJ, 508, L61

Marrone D. P., Moran J. M., Zhao J.-H., Rao R., 2007, ApJ, 654, L57

Melia F., Falcke H., 2001, ARA\&A, 39, 309

Mori K., et al., 2013, ApJ, 770, L23

Nord M. E., Lazio T. J. W., Kassim N. E., Goss W. M., Duric N., 2004, ApJ, 601, L51

Pushkarev A. B., Kovalev Y. Y., 2015, MNRAS, 452, 4274

Rodriguez L. F., Canto J., Moran J. M., 1982, ApJ, 255, 103

Rodríguez L. F., Gómez Y., Tafoya D., 2012, MNRAS, 420, 279

Roy S., 2013, ApJ, 773, 67

Roy S., Pramesh Rao A., 2004, MNRAS, 349, L25

Rybicki G., Lightman A., 1979, Radiative Processes in Astrophysics. A Wiley-Interscience publication, Wiley

Schnitzeler D. H. F. M., Eatough R. P., Ferrière K., Kramer M., Lee K. J., Noutsos A., Shannon R. M., 2016, MNRAS, 459,3005

Shen Z.-Q., Lo K. Y., Liang M.-C., Ho P. T. P., Zhao J.-H., 2005, Nature, 438, 62

Spitler L. G., et al., 2014, ApJ, 780, L3

Wilkinson P. N., Narayan R., Spencer R. E., 1994, MNRAS, 269, 67

van Langevelde H. J., Frail D. A., Cordes J. M., Diamond P. J., 1992, ApJ, 396, 686

This paper has been typeset from a $\mathrm{TEX}_{\mathrm{E}} \mathrm{X} / \mathrm{A} \mathrm{T} \mathrm{E}$ file prepared by the author. 